\title{
Elemental analysis of Chinese red seal inks on xuan paper by ArF laser-induced plume fluorescence
}

\author{
Bruno Yue Cai ${ }^{1 *}$, Vincent Motto-Ros ${ }^{2}$, Vincent Detalle ${ }^{3}$, Judy T.S. Lum ${ }^{4}$, \\ Kelvin S.Y. Leung ${ }^{4}$, Nai-Ho Cheung ${ }^{5}$
}

1 ANA Artwork Material Analysis Company Limited, 1/F Winner Mansion, 691A, Nathan Road, Mong Kok, Hong Kong, China

2 Université Claude Bernard Lyon 1 and the CNRS (UMR5306), Campus LyonTech- La Doua, Bâtiment Kastler, 10 rue Ada Byron, 69622 Villeurbanne CEDEX, France.

3 Centre de Recherche et de Restauration des Musées de France (C2RMF), Louvre Museum, Paris, France

4 Department of Chemistry, Room T1214, Cha Chi-ming Science Tower, Ho Sin Hang Campus, Hong Kong Baptist University, Hong Kong, China

5 Department of Physics, Room T924, Cha Chi-ming Science Tower, Ho Sin Hang Campus Hong Kong Baptist University, Hong Kong, China

* Corresponding author: brunocai@artworkanalysis.com

\begin{abstract}
Chemical analysis of Chinese red seal ink on xuan paper is useful for the authentication of Asian artwork. The analysis has to be nondestructive and has to accommodate artworks of all sizes. We use ArF laser-induced plume fluorescence to analyze six commercial Chinese red seal inks on xuan paper. Two nano-second laser pulses are required for the analysis. The first $355 \mathrm{~nm}$ laser pulse desorbs a thin layer of the ink to create a plume; the second $193 \mathrm{~nm}$ laser pulse induces multi analytes in the plume to fluoresce. Partial-least-square discriminant analysis of the fluorescence spectra unambiguously sorts the six inks while the sampled area is not visibly damaged even under the microscope. The laser probe can handle samples of arbitrary size and shape, is air compatible, and no sample pretreatment is necessary.
\end{abstract}

Keywords: Invisible Damage, Laser plume analysis, Inks

\section{Introduction}

Red seal imprints are present practically on all Chinese paintings and calligraphies. This is a tradition that dates back to the Qin and Han dynasties over two thousand years ago [1]. In general, each artwork is decorated with multiple seal imprints. They signify, respectively, the author, his alias, his studio, the collectors, well-wishes, idioms and various other historic information [1]. This practice is also common in Japan, Korea, Vietnam and Central Asia [2]. Examination of the seal imprints is therefore critically important in 
establishing the provenance and authenticity of Asian artworks.

For a long time, appraisal of seal imprints has been visually performed by experts who focus on the appearance, style and context of the imprints [3]. While this kind of visual examination is very useful, an objective scientific analysis of the inks can be a powerful complement [4].

The ingredients of the Chinese red seal ink are well documented [1]. The making of the ink paste has a long history. By the Yuan dynasty $(\sim 1300 \mathrm{AD})$, the recipe has evolved to the current oil-based version [1]. Typically, the red pigments are fine powder made from minerals such as cinnabar $(\mathrm{HgS})$, minium $\left(\mathrm{Pb}_{3} \mathrm{O}_{4}\right)$, kermesite $\left(\mathrm{Sb}_{2} \mathrm{~S}_{2} \mathrm{O}\right)$ and other shades of red, but modern inexpensive ones may use red dyes instead [1]. The binder is vegetable oil, usually castor oil, which also acts as a dispersant. Fine powder of kaolin $\left[\mathrm{Al}_{2} \mathrm{Si}_{2} \mathrm{O}_{5}(\mathrm{OH})_{4}\right]$, baryte $\left(\mathrm{BaSO}_{4}\right)$ or other clay is added as filler to give body; and ground mugwort fiber is introduced as an absorbent. The oil, powder and fiber are thoroughly mixed to form the paste. Some manufacturers also add preservatives such as borneol and perfumes $[1,5]$.

Red seal ink can be chemically analyzed by a host of techniques. For the organics, chromatographic separation such as thinlayer [6], high-performance [7], and gas chromatography [7], followed by fluorescence or mass spectrometry are very effective methods of analysis. Transmission Fourier-transform infra-red (FTIR) spectroscopy is also useful in identifying specific bonds [8] To perform these analyses, ink samples have to be scraped from the specimen. They are therefore destructive and cannot be applied to the analysis of valuable artworks.

Alternative techniques that are nondestructive have been deployed. Notable examples include micro attenuated-total-reflectance FTIR ( $\mu$ ATR-FTIR) spectroscopy [9], and laser Raman spectroscopy $[6,10]$. Both techniques are sensitive to the vibrational signature of the inorganic pigments [11]. When these vibrational analysis are performed together with elemental analysis, numerous red inks can be sorted unambiguously [8]. Yet once again, the destructiveness of the elemental analysis step is a concern. For example, energy dispersive $\mathrm{x}$-ray (EDX) analysis will require the extraction of ink sample from the artwork for insertion into the analysis chamber [8]. The invasiveness can be reduced if techniques of higher sensitivity are adopted, such as time-of-flight secondary ion mass spectrometry (TOF-SIMS) [12]. When the analyte elements are present at $\mathrm{mg} / \mathrm{g}$ level and higher, the nondestructive portable $\mathrm{x}$-ray fluorescence ( $\mathrm{pXRF}$ ) probe can be used [13].

We have used pXRF to analyze laser printed black toners on photocopier paper and Chinese black ink on xuan paper $[14,15]$. We showed that it is a good screening tool when the analyte elements are abundant (percent range) and when the specimen is optically thick. However, for the elemental analysis of seal imprints on artwork the inked area is likely to be sparse; and for authentication purposes, the signature elements are probably present at low concentrations. In such cases, we have shown that pXRF is best supplemented by an analytical technique that we named plume-LIF, or PLIF for short [14, 15]. PLIF stands for laser-induced-fluorescence of plumes. The plumes are produced by pulsed laser sampling of the specimen. We have demonstrated that PLIF can simultaneously detect multi-elements, including trace ones [18]. It can pin-point down to $\mu \mathrm{m}$ accuracy, causes no visible damage, is 
air-compatible, requires no sample pretreatment, and can accommodate samples of any size [19].

Therefore, PLIF is ideal for the elemental analysis of red seal inks on artworks. In this proceeding, we report such a study that demonstrates the successful sorting of six Chinese red seal inks based on nondestructive PLIF analysis.

\section{Materials and methods}

\subsection{Materials}

Six Chinese red seal inks coded C1 to C6 were selected. They were all acquired from the well-known ink paste manufacturer Xilengyinshe in Shanghai, China. Details are summarized in Table 1. Their prices ranged from entry grade to midrange. C2 and C5 are particularly similar in color. They are chosen to test the discrimination power of our analysis. Each ink was applied evenly on Chinese xuan paper and allowed to air dry in a dust-free environment for two days at room temperature before use. The inked paper was mounted flat on a 1 " $\times 3$ " glass slide for PLIF analysis.

\subsection{PLIF analysis}

The experimental setup for two-laser-pulse PLIF analysis was reported previously [15]. Briefly, the first pulse from a Nd:YAG laser (Continuum Surelite II, $3^{\text {rd }}$ harmonic, 355 $\mathrm{nm}, 10 \mathrm{~Hz}, 9 \mathrm{~ns}$ ) was focused on the sample to desorb a minute amount $(\leq \mathrm{ng}$ ) of the ink mass. The focal spot at the target surface was $25 \mu \mathrm{m}$ radius $\left(1 / \mathrm{e}^{2}\right)$ and the laser fluence was about $200 \mathrm{~mJ} \mathrm{~cm} \mathrm{~cm}^{-2}$. The sample was translated to avoid repetitive ablation of the same area.

$51 \mu$ s later, the desorbed plume was intercepted transversely by the second laser pulse from an ArF laser (GAM Ex5, 193 nm, 10 $\mathrm{Hz}, 8 \mathrm{~ns})$. The ArF laser beam was focused to a point $8 \mathrm{~mm}$ in front of the plume. The spot size at the plume was $640 \mathrm{~mm} \times 280 \mathrm{~mm}$ and its center was $145 \mu \mathrm{m}$ from the sample surface. The ArF fluence was about $440 \mathrm{~mJ}$ $\mathrm{cm}^{-2}$. The ArF laser pulse vaporized the ink particulates in the plume to produce analyte atoms. The trailing portion of the same 193$\mathrm{nm}$ laser pulse induced the various analytes to fluoresce [20].

Fluorescence emissions were collected and imaged onto an optical fiber bundle

Table 1. The Chinese red seal inks and paper used in this study

\begin{tabular}{lll}
\hline Ink & Brand name & Manufacturer \\
\hline C1 & Qinghua quangming zhusha & Xilengyinshe \\
C2 & Qinghua jingmian zhusha & Xilengyinshe \\
C3 & Qinghua meili zhusha & Xilengyinshe \\
C4 & Fencai jianzhu zhusha & Xilengyinshe \\
C5 & Fencai jingzhi shangpin zhubiao & Xilengyinshe \\
C6 & Fencai tezhi zhenpin zhusha & Xilengyinshe \\
\hline & & \\
\hline xuan & Brand name & Manufacturer \\
Ripe & Yuxing fan xuan & Anhui Sheng Jing Xian Yuquan \\
\end{tabular}


whose other end was mounted at the entrance slit of a $0.5 \mathrm{~m}$ spectrometer (Acton SpectraPro-500) equipped with a gateable intensified charge-coupled device (ICCD, Andor iStar DH738F-63). The ICCD was gated on with the firing of the $193-\mathrm{nm}$ pulse and stayed on for $200 \mathrm{~ns}$. The full width of each captured spectrum was $160 \mathrm{~nm}$ and the instrumental resolution was about $1 \mathrm{~nm}$. This resolution was preserved in all off-line spectral smoothing. Three spectral segments centering respectively on 340,500 and 640 $\mathrm{nm}$ covered the whole spectral range used in this study.

The probed area was viewed by an in-line CCD camera in real-time at $64 \times$ magnification to reveal the sample damage caused by each laser shot.

\subsection{AA and ICPMS analysis}

The elemental compositions of the ink pastes and blank xuan paper were analyzed by atomic absorption (AA) and inductively coupled plasma mass spectrometry (ICPMS) to establish analyte concentration for reference. It was performed by adding $7 \mathrm{~mL}$ concentrated $\mathrm{HNO}_{3}$ (Aristar, $\mathrm{BDH}, \mathrm{UK}$ ) to weighed portion of the seal paste. Digestion was carried out in a temperature programmed ETHOS One Advanced Microwave Digestion System (Milestone S.r.l., Sorisole, Italy). After cooling, the digest was diluted to $25 \mathrm{~mL}$ with MilliQ (18.2 M $\Omega \mathrm{cm}$ Millipore, Billerica, MA, USA) water for subsequent elemental determination by ELAN DRC II ICP-MS (PerkinElmer, Waltman, MA, USA) or Spectra AA 220FS Atomic Absorption Spectrometer (Varian, Agilent Technologies, Santa Clara, CA, USA). Elemental content was quantified against calibrated response curves prepared from $1000 \mathrm{gL}^{-1}$ stocks (VHG, Manchester, $\mathrm{NH}$, USA).

\subsection{Chemometrics}

The chemometric analysis was performed using SIMCA (version 14.0).

\section{Results and discussion}

\subsection{PLIF spectra}

We captured more than a thousand single-shot PLIF spectra of the six inks. Fig. 1 shows spectra of inks C1 (black), C2 (blue), C3 (brown), C4 (green), C5 (orange) and C6 (gray) on xuan. The display format is explained in the figure caption. Each trace is the average of 100 single-shot spectra and consists of three 160-nm-wide regions stitched together. The spectral area of each region is normalized to remove shot-to-shot fluctuations. For the purpose of graphical clarity, the vertical scale of the spectra in the $350 \mathrm{~nm}$ to $410 \mathrm{~nm}$ region was shrunk $3 \times$ to keep the strong $\mathrm{Pb}$ I lines within view. The prominent spectral features are labeled by color-coded lines at the bottom. They demonstrated the multi-analyte detection capability of PLIF. None of the spectral features was visible when either laser beam was blocked. This testifies to the synergy of the two laser pulses.

Based on Fig. 1, four observations can be drawn. First, the spectral features among the six inks are similar, making the sorting very challenging. The line identities are given at the bottom of the plot. Lead is from the abundant kermesite. Chromium and antimony are probably from pigments used to shade the red. Aluminum is presumably from the filler. Carbon dimer is from the oil binder. Sodium and calcium are most likely from the mugwort fiber or vegetable oil.

Second, the examination of the fine spectral differences shows that $\mathrm{C} 6$ has the most 


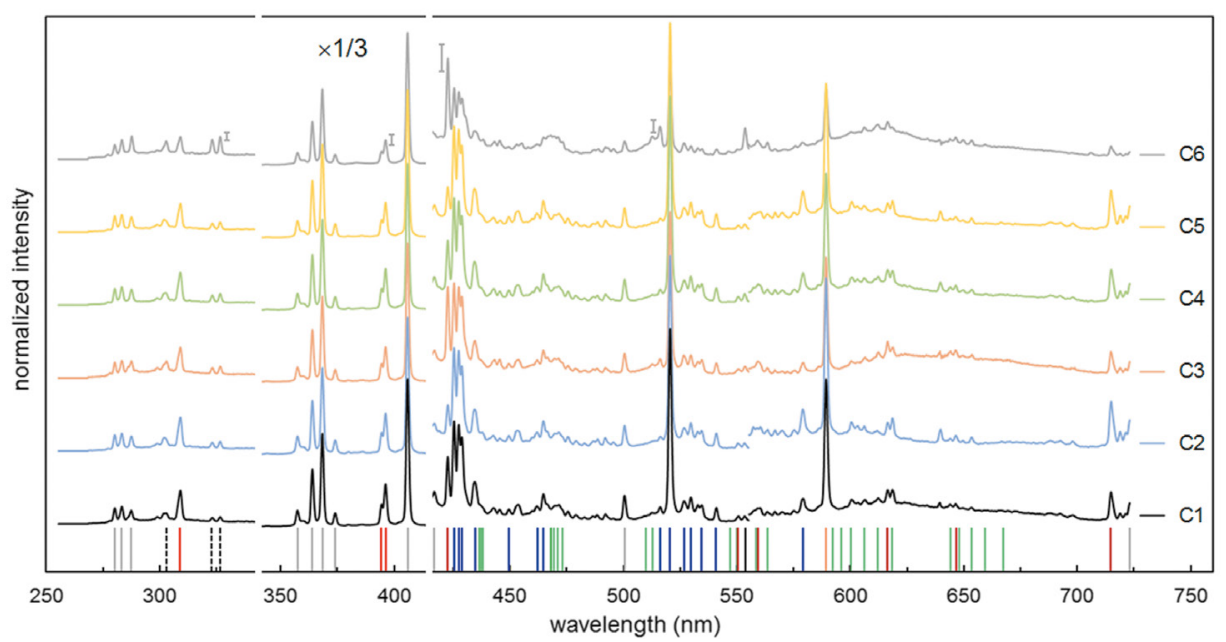

Fig. 1. Normalized PLIF spectra of six red seal inks, C1 to C6. Each trace is the average of 100 single-shot normalized spectra, with the leading pixels zeroed to indicate the baseline. The standard deviations of four selected line intensities of the $\mathrm{C} 6$ trace are indicated by error bars. The traces are offset vertically for graphical clarity. The vertical scale of the $350 \mathrm{~nm}$ to $410 \mathrm{~nm}$ region is shrunk $3 \times$ to keep the strong $\mathrm{Pb}$ I lines within view. Identities of the more prominent spectral lines are color-coded at the bottom: Al I (red), Ba I (black), Ca I (brown), Cr I (blue), Na I (orange), $\mathrm{Pb}$ I (gray), Sb I (black, dotted), and $\mathrm{C}_{2}$ band heads (green).

intense $\mathrm{Sb}$ (323.3 and $326.8 \mathrm{~nm}$ ), Ca (422.7 $\mathrm{nm})$, and $\mathrm{C}_{2}(516.5 \mathrm{~nm}$ band head) features and can be readily sorted. The remaining five inks can then be sorted based on C4 having the brightest Al lines (309.2, 394.4 and 396.2 $\mathrm{nm}$ ) while $\mathrm{C} 5$ the dimmest, and $\mathrm{C} 1$ has the weakest $\mathrm{Sb}$ lines. Then between the last two inks $\mathrm{C} 2$ and $\mathrm{C} 3$, the latter has a significantly brighter Ca line $(422.7 \mathrm{~nm})$. While these distinguishing features are not the brightest spectral lines, their intensities are still many times above the noise and are therefore reliable. This is evident from the relative heights of the error bars drawn against them (Fig. 1, C6 trace).

Third, inks C2 and C5 are not only similar in color, their spectra are also very similar. Careful study shows that $\mathrm{C} 5$ has weaker Al I lines than $\mathrm{C} 2$.

Fourth, the spectral intensities displayed in Fig. 1 do not seem to correlate with the abundance of the elements (Table 2). For example, $\mathrm{C} 6$ has about ten times less lead than $\mathrm{C} 1$ and $\mathrm{C} 2$ but its $\mathrm{Pb}$ I lines are not that much weaker. The discrepancy can be partially explained by the fact that the spectra shown in Fig. 1 are normalized. The intensities are magnified relative to the empirical PLIF spectra by the factors shown in Table 3. As can be seen, the Pb I lines of C6 are magnified about three times more than those of $\mathrm{C} 1$ and $\mathrm{C} 2$. Another complication is the fact that AA and ICPMS analyzed ink pastes rather than ink imprints on xuan. Fractionation of the ink chemicals at the seal imprinting step and during the plume formation process can occur.

\subsection{Chemometric analysis}

Based on the PLIF spectra, we used partialleast-square discriminant analysis (PLSDA) 
Table 2. Concentrations ( $\mathrm{mg} / \mathrm{g}$ ) of the more abundant elements present in the six Chinese red seal ink pastes and the xuan paper used in this study, as measured by atomic absorption (AA) and inductively-coupled plasma mass spectrometry (ICPMS)

\begin{tabular}{lccccccc} 
& \multicolumn{9}{c}{$\mathrm{mg} / \mathrm{g}$} \\
\cline { 2 - 8 } & \multicolumn{9}{c}{$\mathrm{AA}$} & & & ICPMS \\
\cline { 2 - 8 } & $\mathrm{Pb}$ & $\mathrm{Hg}$ & $\mathrm{Cr}$ & $\mathrm{Ca}$ & $\mathrm{Al}$ & $\mathrm{Sb}$ & $\mathrm{Ba}$ \\
\hline xuan & - & 0.004 & 0.006 & 1.34 & 0.578 & - & 0.003 \\
ink C1 & 148 & 6.94 & 18.1 & 7.46 & 2.14 & 0.965 & 0.387 \\
ink C2 & 93.9 & 20.5 & 6.94 & 5.88 & 1.92 & 1.02 & 0.407 \\
ink C3 & 39.8 & 12.0 & 1.75 & 10.6 & 0.981 & 0.457 & 0.995 \\
ink C4 & 57.0 & 44.7 & 8.86 & 13.2 & 1.14 & 7.00 & 1.51 \\
ink C5 & 53.7 & 2.94 & 5.01 & 10.3 & 1.32 & 1.11 & 1.31 \\
ink C6 & 10.7 & 3.09 & 1.29 & 21.3 & 0.444 & 0.208 & 1.18 \\
\hline
\end{tabular}

Table 3. The normalized spectra shown in Fig. 1 are magnified relative to the empirical PLIF spectra. Tabulated are the magnification factors for the corresponding inks for the three respective spectral segments centering at 340,500 and $640 \mathrm{~nm}$

\begin{tabular}{lccc} 
& \multicolumn{3}{c}{ Scaling factor } \\
\hline ink & $340 \mathrm{~nm}$ & $500 \mathrm{~nm}$ & $640 \mathrm{~nm}$ \\
\hline C1 & 1.48 & 0.56 & 1.00 \\
C2 & 1.02 & 0.40 & 1.34 \\
C3 & 2.20 & 0.75 & 1.06 \\
C4 & 1.27 & 0.52 & 1.15 \\
C5 & 1.43 & 0.53 & 1.10 \\
C6 & 3.97 & 0.98 & 1.23 \\
\hline
\end{tabular}

to sort the six inks. For that purpose, we captured on different days two independent sets of spectral data from sample replicates. One set is used to train the PLSDA model while the other set is used to test the model. The training set consists of 200 single-shot panoramic spectra covering the three spectral regions $(1024 \times 3$ pixels $)$ for each of the six inks. In PLSDA language, we have a supervised six-class model with each ink belonging to one class; $1024 \times 3$ variables, $200 \times 6$ training observations. The test set consists of 100 single-shot panoramic spectra for each of the six inks, that is, a total of $100 \times$
6 test observations. The class identity of each of the observations is known.

For data pre-processing, we normalized the total intensity of each 1024-pixel single-shot spectrum to 5,000 to remove the shot-to-shot fluctuations. We then mean-centered the data values for each of the $1024 \times 3$ variables. No outliers are discarded from either the training or the test set.

The sorting accuracy of the $100 \times 6$ test observations is shown in Table 4 in the form of a confusion matrix. As can be seen, the sorting accuracy is $100 \%$ for inks C2, C3 and C6. C4 has the lowest accuracy of $87 \%$. It is mainly confused for $\mathrm{C} 1(8 / 100)$ and $\mathrm{C} 2$ $(4 / 100)$. Overall, the sorting accuracy is $96.2 \%$.

\subsection{Coefficient spectra}

The weight coefficients for the $1024 \times 3$ variables used by the PLSDA model are plotted in Fig. 2 in the form of coefficient spectra. Several features can be noted. First, none of the inks can be unambiguously identified by a single spectral feature. Multiple features have to be weighed. Analytical techniques that can detect multi analytes simultaneously 
Table 4. Confusion matrix of the sorting of six Chinese red seal inks on xuan

\begin{tabular}{|c|c|c|c|c|c|c|c|c|}
\hline \multirow{2}{*}{$\begin{array}{l}\text { Test } \\
\text { class }\end{array}$} & \multirow{2}{*}{$\begin{array}{l}\text { \# test } \\
\text { observations }\end{array}$} & \multicolumn{6}{|c|}{ Model class } & \multirow{2}{*}{ Correct } \\
\hline & & $\mathrm{C} 1$ & $\mathrm{C} 2$ & C3 & $\mathrm{C} 4$ & C5 & C6 & \\
\hline $\mathrm{C} 1$ & 100 & 92 & 4 & 1 & 3 & 0 & 0 & $92 \%$ \\
\hline $\mathrm{C} 2$ & 100 & 0 & 100 & 0 & 0 & 1 & 0 & $100 \%$ \\
\hline C3 & 100 & 0 & 0 & 100 & 0 & 0 & 0 & $100 \%$ \\
\hline $\mathrm{C} 4$ & 100 & 8 & 4 & 0 & 87 & 0 & 1 & $87 \%$ \\
\hline $\mathrm{C} 5$ & 100 & 0 & 1 & 0 & 1 & 98 & 0 & $98 \%$ \\
\hline C6 & 100 & 0 & 0 & 0 & 0 & 0 & 100 & $100 \%$ \\
\hline & & & & & & & avg & $96.2 \%$ \\
\hline
\end{tabular}

are therefore preferred and PLIF is such a technique. Second, the weighed features correspond to the prominent lines of $\mathrm{Pb}, \mathrm{Cr}$, $\mathrm{Ca}, \mathrm{Sb}, \mathrm{Al}, \mathrm{Na}, \mathrm{Ba}$, and $\mathrm{C}_{2}$ identified in Fig. 1. They are also indicated at the bottom of Fig. 2. Third, because mean-centering was used in the data pre-processing, intensities below the mean will be weighed negatively. An example is the Sb I 323.3 and $326.8 \mathrm{~nm}$ doublet of $\mathrm{C} 1$. Fourth, the coefficient spectra of C2, C3 and C6 are less noisy. This is consistent with their high sorting accuracy (100\%). In contrast, the coefficient spectrum of C4 is the noisiest. The corresponding sorting accuracy is also the lowest (87\%).

\subsection{Invisible damage}

We investigated the damage to the sample caused by the $355 \mathrm{~nm}$ laser ablation. We used ink $\mathrm{C} 1$ on xuan as a typical specimen. Micrographs of the craters are shown in

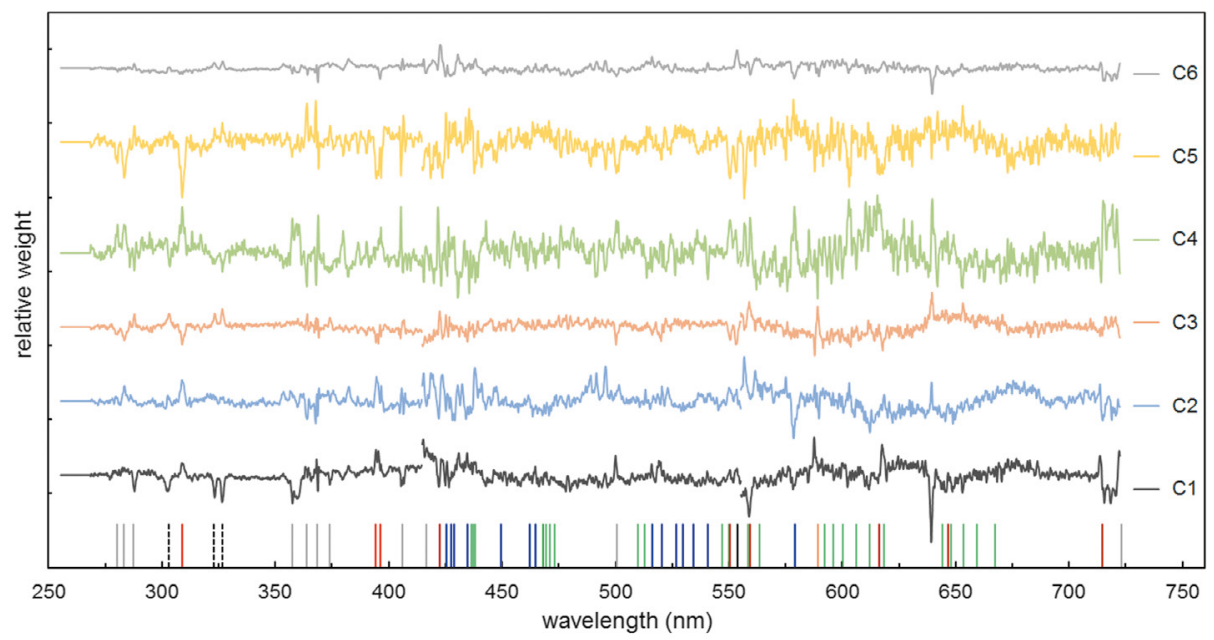

Fig. 2. PLSDA coefficient spectra of the six red seal inks, $\mathrm{C} 1$ to C6. The traces are offset vertically for graphical clarity. The leading pixels of each trace are set to zero to indicate the baseline. Identities of the prominent spectral lines are color-coded at the bottom: Al I (red), Ba I (black), Ca I (brown), Cr I (blue), Na I (orange), $\mathrm{Pb}$ I (gray), Sb I (black, dotted), and $\mathrm{C}_{2}$ band heads (green). 

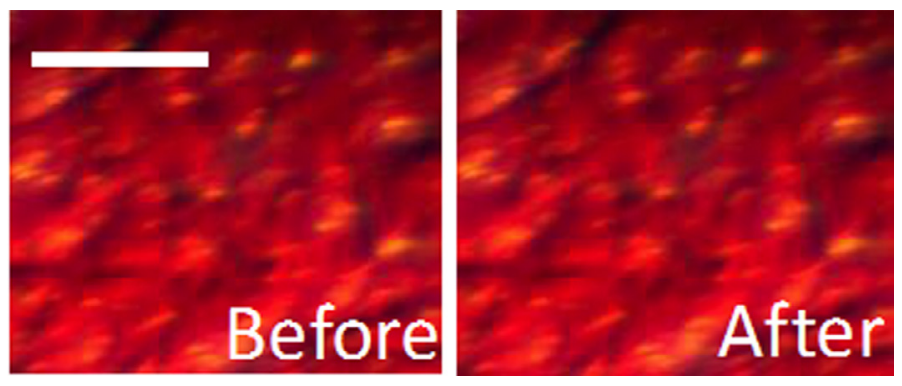

Fig. 3. Micrographs of the craters. The left panel shows the region of interest before the ablation. The right panel shows the same region after ablation by one $355 \mathrm{~nm}$ laser pulse at $200 \mathrm{~mJ} / \mathrm{cm}^{2}$ which is the fluence used in the PLIF analysis. Scale bar represents $100 \mu \mathrm{m}$.
Fig. 3. The left panel shows the region of interest before the ablation. The right panel shows the same region after ablation by one $355 \mathrm{~nm}$ laser pulse at $200 \mathrm{~mJ} / \mathrm{cm}^{2}$ which is the fluence used in the PLIF analysis. As can be seen, damage was not visible even under $64 \times$ magnification.

\section{Conclusions}

We analyzed six commercial Chinese red seal inks that were coated on xuan paper. The inks are from the same manufacturer which is well known in China. Two of the six inks were identical in color and were purposely selected to present analytical challenges. We analyzed the samples by a two-laser-pulse PLIF scheme performed on a high precision optical setup. The first $355 \mathrm{~nm}$ laser pulse ablated a thin layer of the ink; the second $193 \mathrm{~nm}$ laser pulse induced multi analytes in the desorbed ink to fluoresce. Based on the fluorescence spectra, we could tell all the inks apart by partial-least-square discriminant analysis, including the two challenging ink samples. Meanwhile, the sampled area was not visibly damaged even under $64 \times$ magnification.

We plan to extend the PLIF analysis to more seal inks from different manufacturers and different eras in order to establish a comprehensive spectral library. At the same time, we will use pXRF, PLIF and $\mu$ ATR-FTIR to analyze seal inks and to determine the age of the print.

\section{Acknowledgements}

We thank Dr. Xueshi Bai of Centre de Recherche et de Restauration des Musées de France and Dr. Delphine Syvilay of Laboratoire Recherche des Monuments Historiques for useful discussions. This work is supported by ANA Artwork Material Analysis Company Limited under the Hong Kong Innovation and Technology Commission Technology Start-up Supporting Scheme 2016/2017.

\section{References}

[1] S. Deng, Zhuankexue (A study in the art of seal carving), People's Fine Arts Publishing, Beijing 1979. In Chinese.

[2] L. Long, A Ricci Dictionary of Seals, An English and French Dictionary of Chinese Seals and of the Art of Seal Carving, posted by Association Ricci, Paris, http://www.grandricci.org/paraitre_sceaux_leaflet. pdf (accessed 26.10.2016).

[3] Q.Ye, Gu xiyin yu gu xiyin jianding (Ancient seals and how to appraise them), Cultural Relics Publishing House, Beijing 1997. In Chinese.

[4] T. Learner, Analysis of Modern Paints, The Getty Conservation Institute, Los Angeles 2004.

[5] Instructions for the preparation of the red seal ink paste, in Chinese, http://zhidao.baidu.com/ link?url=wm5qRj6i2 cr9hz-GnGNCBS4Pu3_lgJCbGlTfPUClYCSeOo_MkkqJ7N-M7Mbdw0Yn1cD14_ 
DFfWU8DTZLD4chIXf42Vt8GE6itDbUSkDze2S (accessed 26.10.2016).

[6] Raza and B. Saha, "Application of Raman spectroscopy in forensic investigation of questioned documents involving stamp inks", Science and Justice, 53 (2013) 3338, DOI: 10.1016/j.scijus.2012.11.001.

[7] Y.-T. Yao, J. Song, J. Yu et al., "Differentiation and dating of red ink entries of seals on documents by HPLC and GC/MS", Journal of Separation Science, 32 (2009) 291927, DOI: 10.1002/jssc.200900110.

[8] Vila and J.F. Garcia, "Analysis of the chemical composition of red pigments and inks for the characterization and differentiation of contemporary prints", Analytical Letters, 45 (2012) 127285, DOI: 10.1080/00032719.2012.673100.

[9] W. Dirwono, J.S. Park, M.R. Agustin-Camacho, et al., "Application of micro-attenuated total reflectance FTIR spectroscopy in the forensic study of questioned documents involving red seal inks", Forensic Science International, 199 (2010) , DOI: 10.1016/j.forsciint.2010.02.009.

[10] J. Lv, S. Liu, Y.L.J. Feng, Y. Liu and S. Zhou, "Differentiation of inks used for seals by confocal Raman microscopy and Fourier transform infrared microscopy", Spectroscopy, 30 (2015) 33 http://www.spectroscopyonline.com/ differentiation-inks-used-seals-confocal-raman-microscopy-and-fourier-transform-infrared-microscopy?pageID $=1$ (accessed 8.07.2017).

[11] S. Vahur, U. Knuutinen, and I. Leito, "ATRFTIR spectroscopy in the region of $5030 \mathrm{~cm}-1$ for identification of inorganic red pigments", Spectrochimica Acta A, 73 (2009) 7671, DOI: 10.1016/j. saa.2009.03.027.

[12] J. Lee, C. Lee, K. Lee, and Y. Lee, “TOF-SIMS study of red sealing-inks on paper and its forensic applications", Applied Surface Science, 255 (2008) 152526, DOI: 10.1016/j.apsusc.2008.05.094.

[13] F. Rosi, A. Burnstock, K.J. Van den Berg, C. Miliani, B.G. Brunetti, and A. Sgamellotti, "A non-invasive XRF study supported by multivariate statistical analysis and reflectance FTIR to assess the composition of modern painting materials", Spectrochimica Acta A, 71 (2009) 165662, DOI: 10.1016/j. saa.2008.06.011.

[14] P.C. Chu, B.Y. Cai, Y.K. Tsoi, R. Yuen, K.S.Y. Leung, and N.H. Cheung, "Forensic analysis of laser printed ink by $\mathrm{x}$-ray fluorescence and laser-excited plume fluorescence", Analytical Chemistry, 85 (2013) 431315, DOI: 10.1021/ac400378q.

[15] Y. Cai, Z. Huang, M.H.C. Cheung, et al., "Elemental analysis of Chinese black inks on xuan paper by ArF laser-excited plume fluorescence", Analytical Chemistry, 88 (2016) 10970978, DOI: 10.1021/acs. analchem.6b02628.

[16] S.K. Ho and N.H. Cheung,"Minimally destructive and multi-element analysis of aluminum alloys by ArF laser-induced atomic emissions", Journal of Analytical Atomic Spectrometry, 22 (2007) 2997, DOI: 10.1039/B611899B.

[17] S.K. Lau and N.H. Cheung, "Minimally Destructive and Multi-Element Analysis of Steel Alloys by Argon Fluoride Laser-Induced Plume Emissions", Applied Spectroscopy, 63 (2009) 8338, DOI: $10.1366 / 000370209788700973$.

[18] P.C. Chu, W.L. Yip, Y. Cai, and N.H. Cheung, "Multi-element analysis of ceramic and polymeric samples by ArF laser excited atomic fluorescence of ablated plumes", Journal of Analytical Atomic Spectrometry, 26 (2011) 121216 DOI: 10.1039/ C1JA10026B.

[19] Y. Cai, P.C. Chu, S.K. Ho, and N.H. Cheung, "Multi-element analysis by ArF laser excited atomic fluorescence of laser ablated plumes: Mechanism and applications", Frontiers of Physics, 7 (2012) 6778 DOI: $10.1007 / \mathrm{s} 11467-012-0264-x$.

[20] X. Wang, Z. Huang, P.-C. Chu, et al., "The mechanism of ArF laser-induced fluorescence of dense plume matter", Journal of Analytical Atomic Spectrometry, 31 (2016) 236374, DOI: 10.1039/ C6JA00290K. 
